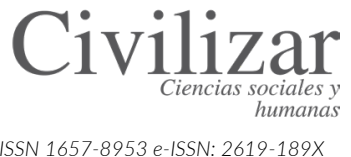

ISSN 1657-8953 e-ISSN: 2619-189X

\title{
Entre la migración y la resistencia en contextos de crisis
}

\author{
Between migration and resistance in crisis contexts \\ Nastassja Rojas Silva
}

* Artículo de reflexión.

§ Doctoranda en Derecho, magíster en Relaciones Internacionales, politóloga mención Ciencias Políticas y Ciencias Administrativas. Profesora universitaria y consultora en temas migratorios, género y democracia, particularmente en el caso Venezuela. Profesional de consultoría en la Escuela de Gobierno Alberto Lleras Camargo de la Universidad de los Andes. (iD) $0000-0001-8568-4138$

Cómo citar:

Rojas Silva, N. (2020). Entre la migración y la resistencia en contextos de crisis. Civilizar: Ciencias Sociales Y Humanas, 20(39), 127-138. https://doi. org/10.22518/jour.ccsh/2020.2ao8

\section{Resumen}

Desde la llegada de la Revolución Bolivariana, la situación del sector educativo venezolano, especialmente el de la educación superior no ha sido fácil. Las políticas en educación superior de la revolución llevaron a un estancamiento de las universidades más reconocidas, sin mencionar el crecimiento de la deserción estudiantil y la situación de las y los profesores a nivel universitario que sigue siendo una de las más precarias en cuanto a ingresos y condiciones de trabajo. Ante este panorama, quienes trabajan en la academia se enfrentan a uno de estos tres caminos: resistir desde las aulas - con los riesgos que ello implica-, renunciar a sus empleos ante la imposibilidad de poder asistir a sus trabajos, o migrar con la esperanza de seguir desempeñándose en este oficio y aportar al rescate de la democracia desde el exilio, lo cual resulta difícil ante los múltiples obstáculos que se presentan. En este sentido, el presente artículo tiene como objetivo reflexionar sobre la migración intelectual venezolana y sus causas, con el propósito de propiciar en futuras investigaciones la discusión sobre los marcos interpretativos que presentan una distinción entre migración calificada y no calificada, por cuanto la situación de este gremio en Venezuela reclama una reinterpretación de los conceptos al encontrarse en una zona gris lejos de la protección internacionales de forma diferenciada.

\section{Palabras clave}

Migración intelectual, profesoras y profesores, educación superior, exilio, Revolución Bolivariana

\section{Abstract}

Since the arrival of the Bolivarian Revolution, the Venezuelan educational sector's situation, especially higher education, has not been easy. The policies in higher education of the revolution led to a stagnation of the most recognized universities. Not to mention the growth of student dropouts and the teachers' position at the university level, which continues to be one of the most precarious in terms of income and working conditions. Against this background, the teaching staff at all levels of training faces one of these three paths: resist from the classroom, with the risks that this implies, resign from their jobs due to the impossibility of being able to attend their jobs, or migrate with the hope of continuing to perform in this profession and contribute to the rescue of democracy from exile, which is difficult given the many obstacles. In this sense, this article aims to reflect on Venezuelan intellectual migration and its causes to foster future research on the interpretative frameworks that present a distinction between skilled and unskilled migration. At the same time, the unions' situation in Venezuela demands a reinterpretation of the concepts as they are in a gray area far from international protection in a differentiated way.

\section{Keywords}

Intellectual migration, teachers, higher education, exile, Bolivarian Revolution 


\section{Introducción a la reflexión de la migración académica}

Aprendí pronto que al emigrar se pierden las muletas que han servido de sostén hasta entonces, hay que comenzar desde cero, porque el pasado se borra de un plumazo y a nadie le importa de dónde uno viene o qué ha hecho antes. Conocí verdaderas eminencias en sus países que no lograron revalidar sus títulos profesionales y terminaron vendiendo seguros de puerta en puerta; también patanes que inventaban diplomas y jerarquías y de alguna manera conseguían colocarse en puestos altos, todo dependía de la audacia y las buenas conexiones.

-Isabel Allende, Paula, 1994

Con estas palabras Isabel Allende habla de su propia experiencia como exiliada de la dictadura de Augusto Pinochet en Chile. Su apellido, su activismo feminista en la televisión y sus columnas críticas le habrían de pasar factura y la obligarían a salir de su país forzada por las presiones y amenazas anónimas. Sin embargo, a pesar de que su destino terminaría siendo la Venezuela pujante de los años 70 -en donde la bonanza petrolera auguraba la esperanza de un nuevo comienzo-, su condición de intelectual, periodista y escritora -más que ayudarla- hicieron difícil su inserción en la sociedad de la época.

El rol de quienes confirman la academia en los procesos de migración adquiere una importancia notable porque, además de que se trata de personas migrantes altamente calificadas, se trata de sujetos que pueden cuestionar desde el extranjero al régimen que los expulsó. Sin embargo, esta capacidad depende en gran medida de su inserción dentro del mercado laboral en las sociedades de acogida. En el extranjero, las condiciones de los académicos varían notablemente. Por un lado, se encuentran quienes logran desarrollar sus competencias académicas con facilidad y se vinculan a universidades, centros de investigación o instituciones públicas o privadas. De otro lado, parte de la academia que en el exilio no logra ningún tipo de vinculación pierde el reconocimiento de otrora y asume roles domésticos sin pago. Adicionalmente, quienes abandonan su rol se dedican a otra actividad que les garantice el sustento y asumen sus nuevas tareas. Finalmente se encuentra el grupo que logra recibir financiación para su sostenimiento por parte de agencias que, desde otros países, apoyan estas formas de resistencia.

El caso de Isabel Allende, pese a su apellido y trayectoria, fue más cercano al de los intelectuales que terminan dedicados a labores diferentes a las imaginadas. Una figura de su talla fue reducida por un gobierno dictatorial a una de las sanciones más viles: renunciar a su actividad profesional, la cual es una consecuencia trágica no solo para los quienes forman la academia, sino también para la sociedad de la época, que pierde la posibilidad de crecer cultural e intelectualmente cuando tienen lugar estos procesos migratorios. Ahora bien, es difícil que todas las personas migrantes logren un espacio en las sociedades fuera de su país, pues muchas veces los países de acogida no cuentan con las condiciones estructurales necesarias.

Con esta reflexión en mente, es necesario examinar la innegable difícil situación económica que atraviesa Venezuela como producto de las condiciones políticas, la cual ha forzado a millones de personas a vivir en el exilio voluntario. La hiperinflación -que no da tregua- y la profunda devaluación de la moneda puso contra la pared a todos los estratos socioeconómicos del país, quienes hoy solo intentan sobrevivir en un país que parece estar hundiéndose ante los ojos de las instituciones latinoamericanas -a veces cómplices- y ante la mirada de una ciudadanía impotente.

Sin querer restarle importancia a las duras condiciones de inseguridad y de inestabilidad política, son probablemente los problemas económicos los que preocupan en mayor medida a la población, porque cuando no hay nada que comer, poco o nada importan las instituciones. Estas dificultades no discriminan y han trastornado la vida profesional, familiar y personal de quienes viven en Venezuela.

De esta compleja coyuntura no se escapa el sector educativo, el cual desde la llegada de la Revolución Bolivariana se caracterizó por una serie de reformas que supuestamente pretendían ampliar la cobertura escolar mediante la política de las Misiones Educativas. Lejos de ello, el sistema educativo ha tenido retrocesos, especialmente el sector de la educación superior, debido a la implementación de nuevas instituciones y al recorte de presupuesto y financiamiento de las universidades estatales tradicionales. Como mencionan Rojas y García (2019), si bien se incrementó la matricula durante los primeros años del siglo XXI, no se ha logrado cumplir con las exigencias de una educación de calidad. Sumado a ello, se impusieron también restricciones a los investigadores para poder visibilizar sus trabajos en los congresos internacionales, así como también 
se redujo el apoyo para realizar postgrados en el exterior.

Carmen García-Guadilla, experta en educación superior comparada, en una entrevista realizada por Gabriela Bronfenmajer (2013), sostuvo que las políticas de educación superior adelantadas durante la Revolución Bolivariana llevaron a un estancamiento de las universidades más reconocidas y productivas, mientras se produjo -al mismo tiempo- una expansión de nuevas instituciones de dudosa calidad. Esta situación -que parece empeorar en el régimen de Nicolás Maduro- ya poco importa; los indicadores y las deficiencias actuales son tanto cuantitativas como cualitativas.

De la mano de la agudización política vino el acelerado declive económico del país como consecuencia de las decisiones desacertadas de los años anteriores, como lo fue la adopción del control de cambio, lo cual creó en una primera etapa -en la práctica- la existencia de dos países en un mismo territorio: uno con unos precios "justos" y que solo vive en la imaginación de quienes ansían poder comer, y otro en donde se transan hasta las cosas más simples bajo el precio del dólar del mercado paralelo. Esta realidad -difícil de entender- trastocó la permanencia de los trabajadores en sus puestos formales, incluyendo aquellos dedicados al oficio de la enseñanza en las universidades, quienes se han visto afectados por las paupérrimas condiciones en materia de infraestructura, la no prestación de servicios públicos -agua potable y electricidad-, el limitado acceso a material bibliográfico actualizado, el desfinanciamiento de la investigación, las dificultades de transporte, entre otros problemas que afectan directamente la actividad académica (Aula Abierta Venezuela, 2020).

Ante las innumerables protestas, el descontento político y el evidente empobrecimiento de la población, el régimen bolivariano ha decretado frecuentemente aumentos sustanciales del salario mínimo, incluso se han dado varios aumentos en un mismo año. Sin embargo, la situación de este gremio sigue siendo una de las más precarias en cuanto a ingresos; además de que esta población está sujeta a las instituciones y al financiamiento por parte de entes externos, con muy pocas posibilidades de ejercer su trabajo de manera independiente, aferrándose a las ruinas del sistema educativo con la esperanza de no perder sus años de dedicación y carrera profesional.
Este panorama llevó a que quienes se dedicaban a la enseñanza e investigación enfrentaran un dilema que cuestiona su vocación y la pone en una balanza frente a la supervivencia propia y de los suyos. Ante un escenario tan desolador que no parece mejorar, el profesorado de todos los niveles de formación ha debido tomar alguno de los siguientes tres caminos. El primero, resistir desde las aulas, con unos riesgos altísimos debido a que pueden terminar siendo presos políticos -como ha sucedido en muchos casos-. El segundo, renunciar a sus empleos ante la imposibilidad de poder asistir a sus trabajos -ya que son mayores los gastos de ir a trabajar que lo recibido en su salario- y dedicarse a la economía informal, sin asegurar con esto el ingreso básico y dejando atrás su carrera profesional. Finalmente, la tercera opción, migrar con la esperanza de seguir desempeñándose en su oficio y aportar al rescate de la democracia desde el exilio, cuestión que es cada vez más difícil ante la devaluación de la moneda, los múltiples obstáculos para poder convalidar los títulos universitarios y la regularización de la situación migratoria en los países de destino.

Esta última opción, la de migrar, resulta una decisión tan difícil de tomar como las anteriores, ya que en alguno profesionales se marchan con un sentimiento de culpa profundo por "abandonar" a sus estudiantes e instituciones y con el temor propio de quien migra. Sin embargo, parece ser el camino de mayor elección en los últimos años. En este sentido, la presente reflexión no pretende centrarse en la dimensión cuantitativa de la migración y de la categorización tradicional, el objetivo es mostrarle al lector que la migración va más allá de las estadísticas sistemáticamente organizadas que, por supuesto, resultan necesarias, pero no suficientes para el abordaje de un fenómeno que se ha complejizado con el tiempo.

Detrás de todos los indicadores que se han presentado en las diferentes líneas de investigación sobre esta problemática, se encuentran personas y familias afectadas y que toman la decisión de migrar, dejando de lado las consideraciones profesionales. Por ello, el propósito principal es presentar una reflexión sobre la migración intelectual venezolana desde la llegada de la Revolución Bolivariana hasta el año 2018 con el fin de identificar las causas que llevaron a esta población a migrar, de acuerdo con una periodización que parece tener una estrecha relación con el contexto económico interno, que 
-con el transcurso del tiempo- los hizo más vulnerables.

\section{Metodología para un primer abordaje del fenómeno}

El presente artículo se encuentra dividido en dos partes desde el punto de vista metodológico: primero, se lleva a cabo una revisión de la literatura académica acerca de la migración intelectual, con un énfasis especial en la búsqueda de producción sobre el proceso vivido por Venezuela a partir de la instalación de la Revolución Bolivariana en el país; segundo, una revisión de la prensa colombiana y venezolana durante el período en cuestión, con un interés especial en el período 2014-2018, a fin de identificar los factores que incidieron en la salida de profesoras y profesores hacia otros destinos. Con este propósito, se realiza un análisis de la informa-

Tabla 1

Revisión de artículos académicos ción recogida en los puntos antes mencionados y se utiliza el programa Atlas.ti para la organización y procesamiento de información cualitativa.

Dicho lo anterior, el proceso de búsqueda de material académico se hizo en inglés y español a través de tres bases de datos y cuatro descriptores diferentes. Con estas restricciones no se cree haber agotado todo el universo de textos académicos, puesto que el principal interés se concentró en artículos publicados en revistas académicas y científicas, dejando de lado libros, capítulos de libro y monografías. La búsqueda incluye, además, trabajos sobre el exilio de académicos del cono sur durante las dictaduras y el exilio de la academia durante el franquismo en España. Sin embargo, aunque estos materiales se referencian, no podría afirmarse que la búsqueda es exhaustiva y logra agotar todos los materiales sobre el tema (tabla 1).

\begin{tabular}{l|c|c|c|c|c|c|c}
\hline & \multicolumn{2}{c}{ Scopus } & \multicolumn{3}{c}{ Scielo } & Redalyc \\
& Buscados & Encontrados & Buscados & Encontrados & Buscados & Encontrados \\
\hline Exilio AND Intelectuales & 4 & 3 & 2 & 0 & 37 & 4 \\
\hline Diáspora intelectual & 2 & 1 & 261 & 10 & 35 & 11 \\
\hline Migración calificada & 7 & 5 & 315 & 13 & 120 & 22 \\
\hline Intelectual AND exile & 6 & 3 & 314 & 9 & 8 & 0 \\
\hline Exilio AND intelectuales & 0 & 0 & 804 & 5 & 0 & 0
\end{tabular}

Fuente: elaboración propia.

Los artículos académicos fueron buscados en Scopus a través de cinco descriptores diferentes; se obtuvo el mejor resultado para efectos del trabajo con 'migración calificada'. Para esta base de datos, no se utilizó ninguna restricción y los resultados fueron más acotados. Los resultados de la base de datos Scielo fueron mucho más amplios y se pudo obtener mayor cantidad de material; sin embargo, el rastreo fue mucho más demorado por la cantidad de documentos obtenidos en cada una de las búsquedas. El descriptor más útil para esta base de datos fue el mismo que para Scopus. En Redalyc fue necesario acotar las búsquedas y dejar que todas las palabras aparecieran pegadas, pues de lo contrario los resultados eran inmanejables. Como resultado, el criterio de búsqueda con mejores resultados coincide también con las otras dos bases de datos.

De esta búsqueda es posible destacar tres aspectos. En primer lugar, la mayor cantidad de biblio- grafía acerca de migración intelectual está relacionada con los procesos de movilidad de académicos e intelectuales de Argentina, Chile y Uruguay durante el período de dictadura. Muchos de estos trabajos se concentran en la inserción de los migrantes y la creación de diásporas en los países de destino. El segundo aspecto está relacionado con la migración de intelectuales españoles durante el período del franquismo. Finalmente, el tercer aspecto está intrínsecamente relacionado con la recepción de migrantes por parte de México, tanto los perseguidos por las dictaduras como aquellos que llegaron durante la purga estalinista.

Pese a los anteriores resultados, se destacan tres vacíos. Primero, no hay trabajos en los que se profundice sobre el rol del profesorado o la migración de quienes se dedican a la investigación, seguramente porque se pasa por alto que estos sujetos altamente calificados -por su condición- no van a 
pasar trabajos o simplemente están en condiciones diferentes a las del resto de la población. El otro vacío que se puede evidenciar está asociado a los tipos de regímenes de los que escapan quienes migran, pues la mayor parte de los textos se concentran en la persecución de gobiernos dictatoriales de derecha. Aunque gobiernos de izquierda como la URRS, la antigua Yugoslavia y la misma Cuba persiguieron a la academia, la literatura es mínima sobre el asunto. Ni hablar sobre el caso de Venezuela, para el que no se encontró un solo artículo publicado en revista científica que hiciera alusión a la migración intelectual, pese al crecimiento exponencial de la literatura sobre migración en la última década. Por último, no se encuentra información que estudie la afectación diferenciada entre mujeres y hombres, que se podría dar en el profesorado, ya que se podría suponer que esto tendría impacto sobre las actividades en las cuales terminan finalmente dedicándose.

La segunda fuente de información estuvo concentrada en la revisión de prensa de los dos periódicos de mayor difusión en Colombia y Venezuela. Para el primer caso, se tuvo en cuenta la página web del periódico El Tiempo, de donde se extrajeron noticias, artículos de opinión e informes de la sección de archivo histórico. Para el segundo caso, se tomó como base el periódico El Nacional, de donde se tomaron únicamente noticias y artículos de opinión. Para los dos portales se tuvo en cuenta toda la información disponible acerca de la migración académica del profesorado. La información extraída de estos portales se complementó con la información de los boletines que, de forma regular, publica la organización Aula Abierta Venezuela.

El período de búsqueda en los dos periódicos se concentró en la tercera etapa migratoria que inicia con las marchas estudiantiles de 2014 y se extiende hasta el año 2018, e incluye el agravamiento de la crisis económica producto de la inflación, la devaluación y el cambio del cono monetario. Los mismos criterios usados para la revisión de la bibliografía fueron usados como descriptores para la búsqueda, incluyendo uno más, a saber: 'migración profesores/profesoras'. Aunque es de interés observar lo que ocurrió con la migración intelectual en toda la región, este trabajo muestra una primera aproximación al fenómeno, tomando como destino Colombia.

Finalmente, la razón por la cual se tomó la prensa como técnica de recolección de informa- ción tiene que ver con la falta de información sobre Venezuela, tanto en términos de indicadores como en fuentes oficiales y bases de datos. La información que se encuentra proviene, en su gran mayoría, de esfuerzos aislados de organizaciones no gubernamentales financiadas por organismos internacionales. No obstante, la información que aquí se proporciona intenta ser lo más fidedigna posible y se toman otras fuentes, como Aula Abierta, organización que tiene como propósito la promoción y defensa de los derechos humanos en el ámbito universitario.

Toda la información recolectada fue sistematizada en el programa Atlas.ti, en donde se crearon dos familias de documentos: una para organizar todos los artículos científicos y académicos y otra para sistematizar todas las noticias, artículos de opinión, informes y boletines. Para cada una de estas familias se realizó una codificación en dos momentos. En el primer momento - a través de la codificación automática- se buscaron los criterios 'salarios', 'persecución' y 'condiciones de vida', para entender las causas de la migración y explicar la masiva salida de cerebros del país. La segunda fase consistió en una codificación abierta en la que se revisaron las citas extraídas de la primera forma de codificación. Es de anotar que no se consiguió información sobre personas académicas y sus destinos, por lo que los códigos se concentraron en las causas y la salida de los migrantes.

Los resultados que se presentan a continuación se muestran con el fin de presentar de forma sistemática y coherente las razones que llevaron a la población referenciada en esta investigación a salir de su país para mejorar sus condiciones de vida. Quienes lean este documento no encontrarán una separación explícita entre la revisión de literatura antes expuesta y la revisión de prensa, sino más bien una construcción narrativa de los procesos que han venido llevando a que la crisis en el sector educativo llegue a condiciones críticas nunca antes vistas en los períodos de dictadura que el mismo país caribeño padeció antes de la Cuarta República.

\section{Breve contexto de la migración venezolana: de la migración por razones políticas a la crisis humanitaria}

Venezuela, como país receptor de migración, tiene una larga historia que se encuentra atada al proceso de desarrollo agrícola y a la nacionalización del sector petrolero en los años 70. Es entre 1999 y 2015 que se presenta una nueva forma de entender 
este fenómeno: la población venezolana empieza a entenderse a sí misma como migrante y a conocer las implicaciones tanto domésticas como internacionales que de ello se derivan. La primera etapa de la migración (2000-2005) estuvo asociada a los despidos de más de 17000 trabajadores de la estatal Petróleos de Venezuela SA (PDVSA) y al retiro de aquellos empresarios que se sintieron en riesgo desde la misma llegada de la revolución. Este hecho provocó la migración de personas altamente calificadas y con solvencia económica, quienes fueron recibidas en toda la región sin ningún tipo de obstáculo ni restricción de los gobiernos (Muñoz, 2018).

La segunda etapa de migración venezolana estuvo relacionada con los cambios políticos producidos desde el 2007 , cuando tomó fuerza el movimiento estudiantil y se hicieron visibles las inconformidades de las universidades con el régimen. Las manifestaciones tuvieron como rasgo distintivo la alta participación de estudiantes de universidades privadas y la voz crítica del sector académico. Los reclamos por parte de estudiantes se intensificaron nuevamente en 2014 con el inicio de manifestaciones en las ciudades de San Cristóbal y Mérida; el 12 de febrero del mismo año, varias ciudades más se unieron. Esta etapa incluyó a la clase media, profesionales y estudiantes, quienes contaban con ciertas condiciones económicas mínimas para establecerse en un nuevo país (Muñoz, 2018).

A raíz del incremento del malestar en más ciudades, se produjo una ola de protestas de gran intensidad que dejó 43 personas muertas, más de 400 heridos y 1853 detenciones, así como la orden de arresto a Leopoldo López -señalado como responsable de los incidentes violentos-. Si bien durante estos siete años el flujo migratorio va a tener variaciones significativas, empieza a ser notoria la llegada de migrantes provenientes de Venezuela a los países vecinos, producto de un período de la crisis caracterizada por el desmejoramiento de las condiciones políticas y económicas del país, pero donde aún muchos sectores seguían en pie manifestando activamente su descontento con el régimen.

La tercera etapa migratoria (2017-2018) -asociada a la llegada de Nicolás Maduro al poder en 2013, pero que no fue otra cosa que el desenlace natural de un cúmulo de malas decisiones económicas y políticas de los años anteriores- es la que va a empezar a causar incomodidades en los países destino de la región, por cuanto aquellos migrantes empezaron a ser población identificada con condiciones vulnerables: mujeres solas o con menores, menores no acompañados, adultos mayores, poblaciones indígenas y una clase media golpeada por la situación de Venezuela (Muñoz, 2018).

Esta última etapa fue la que despertó por primera vez en América una verdadera preocupación al ver colapsados sus sistemas de atención y al evidenciar la limitada capacidad institucional de recepción migratoria de los estados latinoamericanos. Desde el año 2016, uno a uno, cada gobierno de la región se vio en la obligación de admitir la falta de capacidad para administrar y gestionar esta migración, como el mismo gobierno de Colombia lo manifestó en su momento. Ello llevó a que este país vecino buscara cooperación internacional para avanzar en una nueva forma de atención, como lo es el Estatuto Temporal de Protección de 2021.

Como consecuencia de ese colapso e incapacidad, aquellas personas que cruzaban las fronteras venezolanas estaban totalmente desprotegidas. En consecuencia, muchas de estas personas migrantes que en décadas pasadas ejercían oficios como la enseñanza universitaria -y dependían de un salario, especialmente cuando se trabajaba para universidades públicas- se vieron con la necesidad de migrar, pero en unas condiciones muy diferentes a las que pudieron migrar sus pares en el inicio de la crisis. Este grupo poblacional se vio obligado a salir del país sin la posibilidad de contar con los documentos necesarios para ejercer su oficio afuera y sin la solvencia necesaria para iniciar una nueva vida en sus posibles destinos.

Ahora bien, las cifras en cuanto al fenómeno migratorio no son del todo claras y mucho menos se logra encontrar una caracterización detallada de los perfiles migratorios en cuanto a las condiciones de salida y de llegada, ya que -en Venezuela, desde el año 2012- el régimen tomó la decisión de no publicar muchas de las cifras que admitirían una clara deficiencia de modelo económico y político (El Nacional, 2018). No obstante, es necesario resaltar el esfuerzo que han hecho organizaciones como el Alto Comisionado de las Naciones Unidas para los Refugiados- ACNUR y la Organización Internacional para las Migraciones - OIM desde el 2018, al crear la Plataforma Regional de Coordinación Interagencial para Refugiados y Migrantes de Venezuela (R4V) con el fin de mantener las cifras actualizadas y, además, para fortalecer las respuestas nacionales 
y regionales de los gobiernos hacia los refugiados y migrantes de Venezuela.

Actualmente, se estima que hay 5.5 millones de venezolanos migrantes, refugiados y solicitantes de asilo reportados por los gobiernos anfitriones; de ellos, aproximadamente, 4.6 millones están en la región. Es probable que esta cifra sea más alta si se tiene en cuenta que algunas fuentes de los gobiernos no toman en cuenta a la población venezolana con un estatus migratorio irregular (ACNUR, 2021). El país que acoge el mayor número de personas migrantes y refugiadas de Venezuela es Colombia: más de 1.7 millones a diciembre de 2020, además de las que están en tránsito y quienes cruzan la frontera en movimientos pendulares, así como 845000 personas repatriadas colombianas y con doble nacionalidad (Plataforma Regional de Coordinación Interagencial, 2021).

A pesar de las cifras anteriores, y ante la incapacidad para administrar y gestionar esta situación migratoria, Colombia solo había implementado hasta la fecha (2018) la Tarjeta de Movilidad Fronteriza para aquellos que aún vivían en Venezuela, pero necesitaban cruzar constantemente la frontera. El país también concedía el Permiso Especial de Permanencia para la población que había cruzado únicamente por un Puesto de Control Autorizado, olvidando a aquellos que -por uno u otro motivoingresaron a territorio colombiano de manera irregular ante el afán de escapar de la crisis social, económica, política y humanitaria en Venezuela.

Sin embargo, en razón a que el $56 \%$ del total de migrantes venezolanos se encuentra en Colombia en situación irregular -y ante el escenario de que a finales del 2021 podría aumentar la cifra a 6.2 millones de población migrante y refugiada proveniente de Venezuela (Ministerio de Relaciones Exteriores de Colombia, 2021)-, el gobierno colombiano mediante el decreto 216 del 1 de marzo de 2021, aprobó el Estatuto Temporal de Protección para Migrantes Venezolanos Bajo Régimen de Protección Temporal con el fin de implementar un régimen migratorio para que las personas migrantes -tanto en condición de regularidad como de irregularidad- que se acojan a la medida tengan un lapso de 10 años para transitar al régimen ordinario de permanencia en el país.

Las consideraciones presentadas hasta ahora reflejan que la migración venezolana ha puesto sobre la mesa una discusión olvidada en el sistema de protección internacional: la utilidad y flexibilidad de los estándares internacionales ante crisis de diferente naturaleza. El caso Venezuela evidencia que los instrumentos de protección han sido muy rígidos en su comprensión cuando no se habla de una guerra en sentido estricto - ¿cómo y quién protege aquellos que no son perseguidos políticos por nombre y apellido?-, sino de una situación económica que les ha arrebatado a los migrantes hasta la posibilidad de consumir las calorías mínimas diarias requeridas y con la creencia de que por haber tenido en algún momento la posibilidad de acceder a la educación universitaria tienen condiciones suficientes para lograr su supervivencia en un lugar desconocido.

\section{Otro rostro de la migración venezolana: situación de la planta profesoral}

Muy lejos se encuentra de la realidad la versión defendida por los partidarios del régimen bolivariano según la cual, uno de los mayores logros de la revolución son los avances en el sistema educativo. Para ellos, el sistema ha tenido tal éxito que otros países de la región solicitan la llegada de profesionales venezolanos; quienes, debido a su elevado grado de formación, son altamente valorados (Ribas, 2018). La realidad es que el ciclo inflacionario en el país y las precarias condiciones del trabajo formal han sido los detonantes de la renuncia masiva del profesorado de todos los sectores, incluyendo quienes ejercen en las universidades, ya sea para dedicarse a otro tipo de actividades en la informalidad o tomar la decisión final de emigrar. Esta situación resulta paradójica por cuanto, en los años 70, Venezuela gozaba de la mejor reputación en cuanto a calidad académica y condiciones de este gremio. Actualmente la realidad es otra, se tiene el salario más bajo de región, muy por debajo de la media (Rojas, 2005).

De acuerdo con la organización Aula Abierta, según su informe Situación de los profesores universitarios en Venezuela, la deserción notoria y alarmante en este sector se debe, principalmente, a los bajos ingresos (Aula Abierta Venezuela, 2018). Es evidente que tal fenómeno suceda, pues hasta 2003, como método de ajuste salarial para las y los profesores, se aplicaron las Normas sobre homologación de sueldos y beneficios adicionales de los miembros del personal docente y de investigación de las universidades nacionales. Después de esta fecha, los ajustes y congelamientos del salario se han hecho de manera unilateral respondiendo a las condiciones impuestas por el régimen y haciendo los ajustes 
salariales siempre por debajo del índice inflacionario (Aula Abierta Venezuela, 2017a; 2021).

Las condiciones salariales de este grupo se encuentran en estado crítico si se tiene en cuenta que la inflación de enero de 2020 a enero de 2021 fue del $3.478 \%$, sin mencionar las circunstancias en las que tienen que laborar, como son la deserción estudiantil y la falta de insumos para sus clases e investigaciones. Los aparentes esfuerzos del régimen por mejorar la situación del profesorado no resultan ciertos, ya que el aumento de los salarios no se compadece con la devaluación del bolívar. Este gremio ha pasado de tener un ingreso promedio de USD 3000 en 1982 a USD 6.96 en 2018 (Aula Abierta Venezuela, 2021).

Ante este panorama, los y las profesoras venezolanas se enfrentan a tres posibles escenarios, como ya se ha mencionado. Resistir desde las aulas, con unos riesgos altísimos debido a que pueden terminar siendo perseguidos políticos, como ya ha ocurrido. Renunciar a sus empleos ante la imposibilidad de poder asistir a sus trabajos -ya que son mayores los gastos de ir a trabajar que lo recibido en su salarioy dedicarse a la economía informal, sin asegurar con esto el ingreso básico y dejando atrás su preciada carrera profesional. Migrar con la esperanza de seguir desempeñándose en este oficio y aportar al rescate de la democracia desde el exilio, cuestión que es cada vez más difícil ante la devaluación de la moneda, los múltiples obstáculos para poder apostillar sus títulos universitarios y la regularización de su situación migratoria en los países de destino.

Sobre el primer posible escenario, en el año 2017, de acuerdo con el Foro Penal Venezolano (2018), se registraron cerca de 5483 detenciones arbitrarias en el país. Además, según los datos publicados en el Informe preliminar: ataques y represalias contra profesores y estudiantes universitarios en Venezuela (febrero a octubre 2017), dentro de la cifra anteriormente mencionada, cerca de 17 integrantes del cuerpo profesoral y 339 estudiantes habrían sido detenidos de forma arbitraria producto de la divulgación de sus ideas críticas al régimen y por su participación en protestas en reclamación de sus propios derechos. Es aún más preocupante que 8 de estas 17 personas fueron presentados ante la justicia militar, lo cual representa una grave violación de Derechos Humanos.

Uno de los rostros de la presión a este gremio es el del profesor de la Universidad de Carabobo,
Santiago Guevara, quien fue privado de su libertad el 21 de febrero de 2017 y procesado junto a 10 militares ante un Tribunal Militar, acusado de traición a la patria e instigación a la rebelión (Aula Abierta Venezuela, 2017b). El profesor Guevara fue detenido por la Dirección General de Contrainteligencia Militar (DGCIM) por publicar un artículo de opinión titulado De goya a la sana prospectiva en el diario El Boletín de Madrid, España, en cual exponía la situación de Venezuela y el colapso económico del país, producto del modelo económico implantado por el régimen bolivariano (Aula Abierta Venezuela, 2017c).

Tal como lo señala Aula Abierta (2017b), no cabe la menor duda de que su detención se trataba de un caso de violación a la libertad académica y libertad de expresión. Finalmente, Guevara fue excarcelado, junto con otras 43 personas privadas de su libertad por razones políticas, en diciembre de ese mismo año debido a las presiones ejercidas por diferentes instancias internacionales; entre ellas, las realizadas por la CIDH sobre la obligación de garantizar la vida e integridad del profesor. Sin embargo, es importante no confundir estas excarcelaciones con la libertad plena de los presos políticos; se han silenciado muchas peticiones.

En relación con el segundo escenario posible, muchas de las personas solían dedicarse a la enseñanza en educación básica, media y universitaria han decidido cambiar los salones de clase y la investigación por el trabajo informal como una forma de buscar las condiciones mínimas para sobrevivir. Como una forma de protesta, en el año 2015, la profesora María Palmar de la Facultad de Medicina de la Universidad del Zulia decidió salir a la calle a cuidar vehículos a cambio de propinas voluntarias de los ciudadanos, vistiendo un cartel que señalaba "De profesora universitaria a 'Bien Cuidaito" con el propósito de llamar la atención sobre las condiciones indignas del gremio.

Después de 3 horas realizando esta actividad, la profesora corroboró que ciertamente se había ganado más de lo que obtendría por una jornada completa como titular. Como María, son muchos los docentes que decidieron cambiar su oficio de manera forzada debido a la situación económica que agobia a la población venezolana. Sin embargo, como ya se ha mencionado en líneas anteriores, son inexistentes las cifras oficiales al respecto, tan solo existe un reconocimiento tácito de la deserción de la 
planta profesoral, la cual -en palabras del régimen bolivariano- es producto de la guerra económica.

Uno de los cambios realizados por el régimen fue la tabla salarial impuesta en abril de 2020 para ajustar los salarios de las y los profesores, la cual -además de ser una decisión claramente violatoria a la contratación colectiva y los derechos laboraleselimina la carrera académica. Al llegar al máximo escalafón (titular a dedicación exclusiva), una persona no percibe ni para cubrir sus necesidades y servicios básicos, lo que imposibilita vivir dignamente. Por esta razón, la situación salarial mencionada califica a este grupo poblacional en situación de Pobreza Extrema, según lo establecido por Banco Mundial y la Línea Internacional de Pobreza (LIP): perciben menos de USD 1,90 diario.

A continuación, la tabla 2 presenta la escala salarial impuesta por el régimen bolivariano a este oficio con su equivalencia en dólar americano en el mercado paralelo de la fecha de su realización. Es importante considerar los salarios frente al costo de la canasta básica alimentaria. De acuerdo con el Centro de Documentación y Análisis para los Trabajadores (2021), en enero de este mismo año, la canasta básica de alimentos, bienes y servicios tuvo un costo de Bs.S 2.400.00o,oo Así, una familia requiere de 291 salarios mínimos de los bolívares soberanos para poder cubrir sus necesidades básicas.

\section{Tabla 2}

Tabla salarial profesoras y profesores desde abril de 2020

\begin{tabular}{|l|c|c|}
$\begin{array}{c}\text { Escalafón } \\
\text { contratación }\end{array}$ & $\begin{array}{c}\text { Salario dedicación } \\
\text { exclusiva } \\
\text { Bs.S. + cesta ticket } \\
(400 \text { Bs.) }\end{array}$ & $\begin{array}{c}\text { Equivalencia } \\
\text { en dólares } \\
\text { americanos }\end{array}$ \\
\hline Titular DE & $1.934 .270,70$ & 6,96 \\
\hline Asociado DE & $1.757 .761,70$ & 6,32 \\
\hline Agregado DE & $1.601 .559,00$ & 5,76 \\
\hline Asistente DE & $1.463 .626,60$ & 5,26 \\
\hline Instructor DE & $1.340 .997,00$ & 4,82 \\
\hline
\end{tabular}

Fuente: elaboración propia a partir de datos de Aula Abierta (2020).

Como última opción, ante las dificultades económicas para subsistir en el país, parte de esta población -de todos los niveles de formación- ha decidido emigrar. No obstante, quienes lo hicieron en años más recientes tuvieron pocas posibilidades de insertarse en el mismo sector en los países receptores, principalmente por las dificultades y altos costos que representa poder obtener los documentos necesarios para ello. En un escenario optimista, se necesitan de 5 a 12 meses en el proceso para migrar, tiempo en cual se deben obtener los recursos para sobrevivir en el país y recolectar la mayor cantidad posible de dinero para poder costear los gastos de salida, teniendo en cuenta que la moneda se devalúa día a día.

En el año 2017, la presidenta de la FAPUV daba cuenta de la alarmante situación que se vivía, señalaba que "los números de la migración crecen dentro del cuerpo docente mientras que el número de publicaciones académicas disminuye" (Pineda, 2017). En este mismo sentido, Rojas y García (2019) han afirmado que la crisis y falta de apoyo a la investigación, así como la baja competitividad en los salarios, destruyó buena parte del capital intelectual de los grupos de investigación y provocó la fuga de cerebros a otros países.

De acuerdo con los registros de la Federación Venezolana de Maestros, entre 2015 y 2020 más de 100 ooo profesoras y profesores abandonaron el sistema educativo, bien para emigrar del país o para ocuparse en actividades de mayor remuneración (Medina, 2021). Según el Observatorio de Educación FundaRedes (2020), un número significativo de los migrantes son profesionales, muchos de ellos de este gremio, quienes deberían ser prioridad en las políticas que implementa el Estado. Sin embargo, al no ser así, estos profesionales deciden emigrar, lo cual conlleva a dejar un vacío en las instituciones educativas.

Resulta imposible determinar qué porcentaje de la deserción de este grupo estudiado permanece aún en el país y quiénes decidieron migrar, ya que con la ausencia de datos oficiales y ante la creciente migración en condición irregular al pasar la frontera, las personas se convierten en un número registrado por algunas autoridades. No obstante, un estudio de las principales universidades venezolanas, incluida la UCV, asegura que, si la pobreza en Venezuela se encuentra sobre el $87 \%$, la población que sale del país no lo hace con las garantías mínimas necesarias, entre ellos quienes conforman este gremio.

Finalmente, resulta interesante mencionar el análisis realizado por la Organización Internacional para las Migraciones (OIM) -diseñado con base en la Matriz de Seguimiento de Desplazamiento (DTM)-, en el cual se expone el perfil regional de los migrantes y refugiados venezolanos que viajaron 
a 11 países de América Latina y el Caribe durante 2019. El primer grupo incluye a los países fronterizos de Venezuela (Brasil, Colombia, Guyana y Trinidad y Tobago), donde los encuestados eran jóvenes y solteros y tendieron a informar que tenían un bajo nivel educativo, pues la mayoría de ellos solo habían terminado la escuela secundaria. En el segundo grupo de países receptores, Ecuador y Perú, los migrantes también tendieron a ser jóvenes, pero una notable proporción reportaron tener un título técnico o superior. En el grupo final se encuentran aquellos países que están más alejados de Venezuela (Argentina, Chile, Paraguay, Uruguay y Costa Rica), donde más del 9o\% de los encuestados -cuyo promedio de edad fue 36 años- afirmó su voluntad de quedarse en el largo plazo. En los cinco países, se reportaron altos niveles de educación: aproximadamente la mitad o más había completado un grado universitario o un postgrado (Echeverría y Chaves, 2020).

\section{Consideraciones finales}

A partir de la revisión de literatura y con los dispositivos presentados por medio de la exploración de las noticias de prensa, se pone de manifiesto que los marcos interpretativos tradicionales para el tratamiento de los flujos migratorios no resultan eficientes cuando se interpretan como movimientos uniformes que no presentan diferencias claras al venir de un mismo contexto. Esta situación podría ser uno de los factores que interviene principalmente en la ausencia de información específica sobre este gremio. Así mismo, se abre el debate sobre las limitaciones de los estándares internacionales para la protección e interpretación de crisis migratorias cuando no son productos de guerras en sentido estrictico y mucho menos cuando se trata de violación de derechos sociales y económicos, lo cual se observó claramente ante la ola migratoria de los ciudadanos venezolanos y la poca preparación que tenían los países para gestionarla y atenderla.

En cuanto al fenómeno propiamente de la migración venezolana y, particularmente, de quienes conforman la planta universitaria, se concluye que no se trata de una tradicional diáspora intelectual en el exilio, como sucedió con quienes huyeron de las dictaduras del cono sur e incluso con los exiliados españoles. Este tipo de diásporas solo son posibles cuando se dan en grupos reducidos y en donde las persecuciones ocurren con nombre propio o se cuenta con el capital migratorio suficiente para lograr subsistir o, al menos, contar con la capacidad de llegar a un tercer Estado que asegure la protección que el sistema internacional ha planteado como ideal.

La migración venezolana de este gremio presenta ciertas particularidades en comparación con las demás crisis migratorias conocidas hasta el momento en la región, especialmente en cuanto a cinco aspectos:

Cuestionan la asociación directa de la migración calificada y el aseguramiento de inserción en el país de llegada, ya que, desde el mismo proceso realizado con la legalización de sus títulos profesionales para el ejercicio de sus actividades, la migración resulta cada vez más difícil. Por tanto, no existe una relación directa entre su nivel de formación y facilidades que podrían tener en la sociedad de acogida.

No es posible hacer un trazado claro de las trayectorias migratorias de estas personas. Por tanto, se desconocen las condiciones de vida reales de cada uno de los migrantes. Si bien las universidades reportan la pérdida y fuga de cerebro -dadas las condiciones laborales-, ante la inexistencia de registros migratorios en Venezuela, se desconocen las cifras de movilidad y el estatus actual de esta migración.

El sistema internacional de protección deja por fuera a aquellos migrantes que son amenazados por condiciones socioeconómicas. Llama la atención que en contextos de crisis como el venezolano, quienes deciden emigrar se encuentran en condición de vulnerabilidad más allá de sus condiciones en el lugar de origen; en consecuencia, surge una tardanza de medidas para la protección especial.

Una alta calificación profesional no asegura redes ni procesos efectivos de inserción laboral, más aún cuando se trata de crisis extendidas en el tiempo y las condiciones de la movilidad misma son cada vez más precarias.

Ante contextos de crisis, la migración calificada puede pasar a ocupar y reclamar la misma atención y protección de la migración no calificada, debido a que -en condiciones como las descritas en este texto- la realidad de quienes migran no se encuentra dentro de los perfiles tradicionales o incluso de aquellos caracterizados en las primeras etapas migratorias provenientes de Venezuela.

Por último, con la reflexión presentada en esta investigación, como un primer acercamiento 
a la situación particular de las universidades y de quienes conforman su planta, valdría la pena preguntarse por la afectación particular que esto trae al desarrollo del mismo conocimiento y estudio de diferentes disciplinas.

\section{Referencias}

ACNUR. (2021). Refugiados y migrantes de Venezuela. Plataforma de Coordinación para Refugiados y Migrantes de Venezuela. https://r4v.info/es/situations/platform

Aula Abierta Venezuela. (2017a). Deserción Universitaria en Venezuela. http://aulaabiertavenezuela.org/ wp-content/uploads/2017/12/AULA-ABIERTA-VENEZUELA-INFORME-PRELIMINAR-DESERCI\%C3\%93N-UNIVERSITARIA-EN-VENEZUELA.pdf

Aula Abierta Venezuela. (2017b). Informe preliminar: Ataques y represalias contra profesores y estudiantes universitarios en Venezuela (febrero a octubre 2017). http://aulaabiertavenezuela.org/wp-content/uploads/2017/08/05-2018-Restricciones-y-represalias-contra-estudiantes-y-profesores-universitarios-en-Venezuela-Febrero-Julio-2017.pdf

Aula Abierta Venezuela. (2017c). Profesor Santiago Guevara es liberado luego de 10 meses de su arbitraria detención. http://aulaabiertavenezuela.org/index. $\mathrm{php} / 2017 / 12 / 24 /$ profesor-santiago-guevara-es-liberado-luego-de-10-meses-de-su-arbitraria-detencion/

Aula Abierta Venezuela. (2018). Situación de los profesores universitarios en Venezuela. http://aulaabiertavenezuela.org/wp-content/uploads/2017/o8/ Informe-profesores-29062018.pdf

Aula Abierta Venezuela. (2020). Informe preliminar: Afectaciones a la educación de calidad en las universidades públicas venezolanas en el marco del COVID-19. http://aulaabiertavenezuela.org/wp-content/ uploads/2020/o4/AFECTACIONES-A-LA-EDUCACI\%C3\% $\% 3 \mathrm{~N}-D E-C A L I D A D-E N-L A S-U N I-$ VERSIDADES-P\%C3\%9ABLICAS-VENEZOLANAS-EN-EL-MARCO-DEL-COVID-19-1.pdf

Aula Abierta Venezuela. (2021). Situación del derecho a la libertad de asociación y de expresión de los profesores universitarios en Venezuela [Informe preliminar].

Bronfenmajer, G. (2013). Entrevista: Carmen García Guadilla. Tres décadas dedicadas al estudio de las universidades. Cuadernos del Cendes, 31(85), 123134 .

Centro de Documentación y Análisis para los Trabajadores. (2021). Canasta alimentaria, enero de 2021. http://cenda.org.ve/

Echeverría, C., y Chaves, D. (2020). Un perfil regional de los migrantes y refugiados venezolanos en
América Latina y el Caribe. Migration Policy Institute. https://www.migrationpolicy.org/research/ perfil-regional-migrantes-refugiados-venezolanos-america-latina-el-caribe

El Nacional. (2018). Voces y cifras del éxodo venezolano en América Latina. El Nacional. https://www.elnacional.com/gda/voces-cifras-del-exodo-venezolano-america-latina_250220/

Foro Penal Venezolano. (2018). Reporte sobre la represión en Venezuela en 2017. https://foropenal.com/ reporte-la-represion-venezuela-2017/

Medina, V. (2021). El sistema educativo venezolano en terapia intensiva. Debates IESA. http://www.debatesiesa.com/el-sistema-educativo-venezolano-en-terapia-intensiva/

Ministerio de Relaciones Exteriores de Colombia. (2021). ABC Estatuto Temporal de Protección-Migrantes Venezolanos.

Muñoz, B. (2018). Diáspora venezolana: Tres olas migratorias en 20 años. El Nacional. https://www. elnacional.com/opinion/columnista/diaspora-venezolana-tres-olas-migratorias-anos_262845/

Observatorio de Educación FundaRedes. (2020). Informe de educación. FundaRedes. https://www. fundaredes.org/informes/Educativo-Informe-2020-obs-educacion.pdf

Pineda, J. (2017). Éxodo de docentes e investigadores es un «golpe bajo» para la educación pública. Efecto Cocuyo. https://efectococuyo.com/la-humanidad/ exodo-de-docentes-e-investigadores-es-un-golpe-bajo-para-la-educacion-publica/

Plataforma Regional de Coordinación Interagencial. (2021). RMRP 2021 for refugees and migrants from Venezuela (pp. 95-119). ACNUR y OIM. https:// rmrp.r4v.info/

Ribas, C. (2018). La migración en Venezuela como dimensión de la crisis. Pensamiento Propio, 47, 91128.

Rojas, R. (2005). Historia de la Universidad en Venezuela. Revista Historia de la Educación Latinoamericana, 7, 75-100.

Rojas, R., y García, J.P. M. (2019). Políticas educativas en Venezuela (2000-2016): Una mirada crítica. Revista Historia de la Educación Latinoamericana, 21(32), 155-192. 